retired professor of natural philosophy at Glasgow, who had quarrelled with Sanders. The note contains the expansion of an elliptic integral in a doubly infinite series and solves the finite motion of a circular pendulum. In 1674 Gregory was appointed to the chair of mathematics in Edinburgh, which he only held for a year before he died. He married in 1669 Mary, daughter of George Jamesone the painter, and widow of Peter Burnet, of Aberdeen, and they had two daughters and a son, James, afterwards professor of physic in King's College, Aberdeen (d. 1731).

A long correspondence (1668-1675) between Gregory and Collins has happily been preserved. Gregory's letters were printed in Rigaud's "Correspondence of Scientific Men of the 17th Century". Collins's letters, which are now in the University Library at St. Andrews, have never yet been published, but the present tercentenary year affords an appropriate opportunity for doing so, particularly as they contain-on the blank spaces of the letters-some fifty mathematical notes of James Gregory in his own hand, revealing either the methods employed in such results as he sent to Collins by letter, or in still newer work which never saw the light. Careful scrutiny, begun in 1932, has unravelled their contents, and shows that Gregory actually anticipated Taylor, by forty-five years, in his famous expansion theorem, that Gregory could integrate $\sin ^{n} \theta$, and had gone deeply into the theory of rational right-angled triangles and Diophantine quadratic equations. It is now possible to judge how slight was his mathematical contact with Newton; also how Barrow and Gregory influenced one another. It is noteworthy that Gregory probably never even heard of the name of Leibniz. These discoveries among the old letters go far to explain the fact that in his day Gregory was held to be second only to Newton.

\title{
Vaccination of Cattle against Foot-and-Mouth Disease
}

$\mathrm{T}$ HE devastating epizootic of foot-and-mouth disease which has raged over Western and Central Europe amongst cattle, sheep and pigs since last autumn has by no means come to an end, though there have been some signs of local or temporary abatement in those areas first invaded in Holland, Belgium and parts of France and Germany. Sweden, Norway and Denmark have suffered little, and are always ready to stop early invasion by slaughter. In Great Britain there have been exceptionally severe and numerous outbreaks, but the number of farms affected and of animals infected or lost by slaughter has not approached that of the Continental calamity. On June 1 last, the number of farms infected in Germany was officially reported as 42,306.

Persistent research work in Germany has been carried out for forty years almost continuously, and since the Great War by Waldmann on the island of Riems ; in France much valuable work was also done by Vallée at Alfort. In Great Britain research has been in progress since 1924, but though knowledge of the disease has increased, it has only been possible to increase the means of control in special circumstances and to reduce the spread of infection. Experimental stations have also been set up in recent years in Holland and Denmark.

The chief aim of investigation has been to find a method of protective vaccination, and many attempts have been made to devise a safe vaccine made from the virus, attenuated by biological, chemical or physical treatment, or made safe by the manner of its administration.
Inactivation of the virus by formalin has until recently appeared to be the most hopeful method, and has perhaps never been tried in the field with sufficient care and persistence. During the last few years, a technique of adsorption of virus with aluminium hydroxide has been very carefully worked out with great detail by Schmidt and his colleagues in Denmark on guinea pigs, and very promising results have been published. He attached great importance to the administration of the adsorbate-vaccine subcutaneously, for by other routes it was capable of producing infection. The results of vaccination given by Schmidt closely resemble those obtained with formalinized vaccine, but the adsorbate appears to be less safe and to give a somewhat higher degree of protection, as might be expected from a less completely inactivated virus. Waldmann maintains that Schmidt's vaccine is too dangerous, and he does not accept the suggestion that the active virus can only be released so slowly from the adsorbate as to be safe though still capable of giving active immunity.

A modification of Schmidt's vaccine has recently been used by Waldmann in Germany with additional safeguards to ensure its inability to produce the disease and spread infection. It was first tested on cattle at the Riems station with good results. The accessory chemical or physical means of attenuation devised by Waldmann have not yet been published, though an account of the vaccination of more than 40,000 cattle with this preparation has appeared in the Berliner tieräratlicher Wochenschrift, Nos. 22 and 24 (1938). 
The initial difficulty in vaccination so as to prevent an invasion of the disease has always been that the vaccine must be prepared from active virus, and only relatively small quantities of virus can be obtained from one infected cow.

The immense number of infected animals in Germany at the present time, and the high degree of organization in the collection of material in outbreaks in separated districts, and in the preparation of the vaccine from the virus, have enabled this relatively large trial to be made in that country. The hope expressed that it will be possible to cultivate in vitro large quantities of suitable virus has so far not been realized. The results of the campaign reported from the areas dealt with appear to be good. Vaccine has been given to more than 40,000 cattle with apparently no spread of infection from the vaccinated animals. It is claimed that almost 100 per cent of the vaccinated animals have developed immunity within fourteen days and some degree of resistance in five days. There is also some evidence that the immunity lasted two to three months at least. Sheep, goats and pigs were not vaccinated as they were considered much less susceptible than cattle, and few outbreaks in these animals are mentioned in the areas where cattle were vaccinated. Three different groups of animals were vaccinated :

The first group of 9,582 normal cattle were in an uninfected district and were isolated for ten days to test the inability of the vaccine to infect normal cattle. The cattle were in thirty-three villages (950 farms) and were vaccinated between March 8 and April 9. No instances of the vaccine producing the disease were observed; only six cases of infection occurred, and there appeared to be good reason to attribute these to other sources. The animals also presumably developed a good immunity, as they remained free from the disease in the neighbourhood of an advancing epizootic.

The second group consisted of 19,486 normal cattle in districts where already a single outbreak had occurred; in this group, though a number of infections occurred in the first fortnight after vaccination, fresh cases then ceased and the animals appeared to have become immune.

The third group of 11,608 cattle was in districts where several outbreaks had already occurred and many of the animals had received an anti-serum. The number of cases after vaccination here was large, but fresh cases did not appear after the twelfth to fourteenth day, and this immunity was attributed to the vaccine. The districts for vaccination were selected because they were in the line of advance of the epizootic. It is stated that even eight days after vaccination the cases of disease appearing were fewer and less severe, and after fourteen days the immunity was almost complete.

In this field experiment there were no exact 'controls', and the course which the epizootic would have taken in the absence of vaccination could be inferred only from the events in the early days after vaccination and records of its course in other unvaccinated farms; the latter details are not included in the published account. It is possible that the synchronous and parallel outbreaks in other herds afforded sufficient 'controls', but the evidence has not appeared.

\section{The Scophony Television Receiver}

\section{By H. W. Lee, Scophony Laboratories}

$\mathrm{T}$ HE Scophony television receiver is an opticomechanical system and possesses the advantages over the purely electric systems employing a cathode ray tube that (1) larger pictures are possible without great expense; (2) high voltages are not necessary-the voltage of a radio set is sufficient; (3) there is lower power consumption for a given picture size. It is scarcely necessary to add that the Scophony receiver works on the B.B.C. transmission.

The essential parts of an optico-mechanical television receiver are: (I) optical elements for producing an illuminated area on the screen; (2) means for moving the illuminated area over the screen; this is at present done by giving it two components of uniform motion in two directions at right angles, one horizontally and one vertically ; (3) means for modulating the intensity of the illumination in accordance with that of corresponding areas of the picture to be reproduced. The modulated signals are received in the form of electro-magnetic waves and are converted into variations in voltage in an electrically tuned circuit; these have to be converted into variations in light intensity. The difficulty of this conversion arises from the high frequency of the signals, which is $5 \times 10^{8}$. (The picture is divided up into approximately $500 \times 400$ elements and scanned 25 times a second.) In sound recording, the highest frequency 